\title{
Competencias Transversales: Percepción de su desarrollo en el Grado en Ingeniería en Diseño Industrial y Desarrollo de Productos
}

\author{
J. Enrique Agudo, Remedios Hernández-Linares, Mercedes Rico y Héctor Sánchez \\ Universidad de Extremadura, Centro Universitario de Mérida, Avda. Santa Teresa de Jornet, 38, \\ 06800 Mérida, Badajoz-España. (e-mail: jeagudo@unex.es, remedioshl@gmail.com, mricogar@unex.es, \\ sasah@unex.es.)
}

Recibido May. 28, 2013; Aceptado Jul. 05, 2013; Versión final recibida Jul. 19, 2013

\begin{abstract}
Resumen
Se realizó una investigación con estudiantes del Grado en Ingeniería en Diseño Industrial y Desarrollo de Productos de la Universidad de Extremadura en España durante dos años consecutivos para evaluar la adquisición de competencias transversales. El estudio se enmarca en el contexto del nuevo sistema de enseñanza basado en competencias que reta a los docentes a buscar estrategias para enseñar y evaluar dichas competencias. Los datos fueron analizados estadísticamente tras un proceso de remuestreo, Bootstrap. En general, el alumnado de segundo curso presenta una mejor percepción del desarrollo de las competencias transversales que el de primero, especialmente en las competencias de trabajo en equipo, conocimiento de las características personales y aptitudes profesionales y capacidad para conseguir los objetivos programados. La principal aportación de este estudio consiste en emplear herramientas para evaluar al alumnado universitario de forma integral, considerando las competencias transversales como parte fundamental del proceso de formación.
\end{abstract}

\section{Generic Competences: Competence Development in Students of the Bachelor of Engineering in Industrial Design and Product Development}

\begin{abstract}
A study was performed to evaluate the acquisition of generic competences by a group of students of the Bachelor of Engineering in Industrial Design and Product Development of the University of Extremadura in Spain. The study is done in the context of the new higher educational system based on competences which challenges educators to devise new strategies to teach and assess the development of those competences. Data were statistically analyzed using a re-sampling technique, Bootstrap. The results show that the second-year students tend to demonstrate better perception of their competence level than first-year students, among which team work, a deep insight on personal and professional features and the ability to reach the fixed objectives stand out. The main contribution of the study consists of emphasizing the use of tools to evaluate the university students in an integral form, considering transversal competences as a fundamental part of the educational process.
\end{abstract}

Keywords: competency based education, higher education, engineering education, transversal competences, European Higher Education Area 


\section{INTRODUCCIÓN}

La globalización de los mercados, la internacionalización empresarial, la creciente rapidez con que se producen los cambios políticos, sociales, tecnológicos y económicos exigen a los futuros profesionales no sólo la adquisición de conocimiento teórico, sino también el desarrollo de ciertas habilidades y competencias. Estamos viviendo una nueva realidad en la que ganan protagonismo conceptos como el de empleabilidad, que pone de manifiesto la necesidad de armonizar los perfiles académicos de los graduados con los perfiles profesionales requeridos (Alonso et al., 2009). La Universidad no puede dar la espalda a esta nueva y cambiante realidad, y se enfrenta al reto de crear un tejido empresarial de alto nivel, de proporcionar oportunidades al estudiante para desarrollar las competencias que demanda un mercado laboral cada vez más competitivo, y una sociedad cada vez más dinámica e innovadora (Cajide et al., 2002; Ortega, 2010). La estrategia del proceso de convergencia europea, a través del Espacio Europeo de Educación Superior (EEES), ha tratado de hacer frente a este desafío basándose en el enfoque o desarrollo de competencias.

Este nuevo enfoque supone un cambio de paradigma metodológico, y nos obliga a los docentes a articular mecanismos que nos permitan determinar si el alumnado posee las competencias recogidas en el título al finalizar sus estudios. En el caso de las competencias específicas, que son aquellas relacionadas directamente con el puesto de trabajo (Solanes et al., 2008), el desafío parece accesible. Sin embargo, la tarea se torna verdaderamente compleja al hablar de competencias transversales, entendiendo por éstas a las que hacen referencia a atributos personales de carácter cognitivo, social, actitudinal o valorativo, enriqueciendo el comportamiento profesional y añadiendo valor al empleado (Corominas, 2001).

Ante tal dificultad, un grupo de docentes del Centro Universitario de Mérida de la Universidad de Extremadura decidimos embarcarnos en esta investigación con un doble objetivo. Por un lado, tratar de aplicar un cuestionario que nos permitiese conocer la opinión del alumnado de primer curso del Grado en Ingeniería en Diseño Industrial y Desarrollo de Productos (en adelante, GIDIDP), sobre el nivel de competencias transversales adquirido, para así poder detectar en cuáles de ellas manifestaban tener más carencias y por tanto, en cuáles deben incidir más los docentes para desarrollarlas adecuadamente. Por otro lado, otra de las metas era intentar analizar si la percepción de estos mismos estudiantes sobre sus competencias había o no cambiado tras un curso académico para evaluar así nuestra propia actuación docente, y detectar las competencias transversales en las que el alumnado perciba haber progresado menos, y en las que, por tanto, será necesario insistir en próximos cursos. Para alcanzar dichos objetivos se realizó un estudio con el alumnado de dicha titulación, estudio que fue llevado a cabo en dos años consecutivos, correspondientes con los cursos académicos 2010/2011 y 2011/2012.

\section{MARCO TEÓRICO}

La Declaración de la Sorbona en 1998 supuso el primer paso para la construcción del Espacio Europeo de Educación Superior. Desde entonces ha ganado protagonismo el concepto de empleabilidad, entendido como la aptitud de encontrar un trabajo en relación a las cualificaciones que uno tiene o la relación entre la educación y la vida profesional de cada persona (Alonso et al., 2009). Cada vez más las empresas valoran y evalúan a sus empleados a través de competencias, lo que parece confirmar que tal y como propuso McClelland (1973), el nivel de competencia constituye un diferenciador crítico del rendimiento (Boyatzis, $2008,5)$. Además la propia Organización para la Cooperación y el Desarrollo Económico (OCDE, 2001, 1) recoge que "el desarrollo sostenible y la cohesión social dependen en gran medida de las competencias de toda nuestra población".

Todo ello justifica la existencia de un claro interés por facilitar un cambio en el sistema de Educación Superior para hacer frente a la globalización y contribuir a la llamada sociedad del conocimiento (Ponsa, et al., 2009). Las Universidades de toda Europa tienen la mirada puesta en la inserción laboral de sus egresados. $Y$ dado que un egresado universitario es tanto más empleable cuando mayor es su capacidad para adaptar sus competencias a las demandas del mercado laboral, las Universidades han de enfrentarse al reto que supone formar titulados universitarios con competencias que les permitan añadir valor y afrontar con éxito el ejercicio de sus respectivas profesiones.

En la Unión Europea, desde hace unos años, se está prestando mucha atención al concepto de competencia (Mulder et al., 2009), que ha adquirido una importancia notable en el discurso analítico de la empleabilidad (Alonso et al., 2009). Las competencias facilitan el desarrollo de una verdadera educación integral al englobar todas las dimensiones del ser humano (saber, saber hacer y saber ser y estar), y suponen un referente para la superación de una enseñanza meramente academicista. 
Así, el EEES ha adoptado el enfoque de competencias como base para la confección de los nuevos planes de estudio en los diferentes estados miembros de la Unión Europea. En el caso concreto de España, el Real Decreto 1393/2007, de 29 de octubre, por el que se establece la ordenación de las enseñanzas universitarias españolas, recoge explícitamente que "los planes de estudios conducentes a la obtención de un título deberán (...) tener en el centro de sus objetivos la adquisición de competencias por parte de los estudiantes, ampliando, sin excluir, el tradicional enfoque basado en contenidos y horas lectivas". En este mismo sentido, un año después, el Protocolo de evaluación para la verificación de títulos universitarios oficiales (grado y máster) de la Agencia Nacional de Evaluación de la Calidad y Acreditación (ANECA) insiste en que el título debe detallar las competencias que el estudiante ha de haber adquirido al finalizar sus estudios.

Aunque existen muchas definiciones del término competencia, con variados matices (Jiménez et al., 2012), de acuerdo a la Organización para la Cooperación y el Desarrollo Económico (2011), una competencia es más que conocimiento y habilidades. Incluye la habilidad para reunir demandas complejas, haciendo uso y movilizando recursos psicosociales (incluyendo habilidades y actitudes) en un contexto particular. En esta misma línea Rychen y Salganik (2000) manifiestan que las competencias son la habilidad para reunir demandas complejas, movilizando recursos psicológicos y cognitivos. En el contexto educativo, las competencias son una combinación de conocimientos, habilidades y actitudes que describe los resultados de aprendizaje de un programa educativo (Rico et al., 2013). De acuerdo con el Informe Delors (Delors, 1996) no conviene limitarse al mero aprendizaje de un oficio, sino que es necesario adquirir una competencia que permita hacer frente a numerosas situaciones, algunas imprevisibles. Es decir, además de las competencias específicas, es necesario que el estudiante adquiera una serie de competencias transversales que pueden ser comunes a cualquier titulación (González y Wagenaar, 2003).

Las competencias pueden ser clasificadas en específicas y transversales. Las competencias específicas son las relativas a las habilidades técnicas necesarias para desempeñar con éxito la actividad o el trabajo relacionado con la cualificación. Tales competencias cambian más frecuentemente que las competencias transversales, y pueden dividirse en conceptuales, procedimentales y profesionales. Las competencias transversales son aquellas que, si bien no están directamente relacionadas con el conocimiento técnico, se requieren para aplicar dichas habilidades técnicas a situaciones diferentes. En el contexto actual, caracterizado por una cambiante economía intensiva en información, los trabajadores deben poseer habilidades transversales, disposiciones y atributos que sean transferibles a muchas situaciones y áreas laborales (Bridgstock, 2009; Rico et al., 2013). Evidentemente no se puede dar la espalda a las competencias específicas o profesionales, pero es cierto que competencias específicas consideradas importantes en un momento dado pueden quedar obsoletas poco después. Sin embargo, todo individuo necesita desarrollar una serie de competencias transversales para su propia realización personal, para poder ser ciudadanos críticos, para facilitar su inclusión social y para encontrar empleo. Las competencias transversales incluyen, pues, competencias intelectuales, personales, interpersonales, organizacionales y empresariales, y se consideran fundamentales para capacitar al estudiante a trabajar en diversos ámbitos profesionales, así como para integrarse con éxito en la vida social (Ortega, 2010) (tabla 1).

La evaluación de las competencias es una de las tareas más importantes en el proceso formativo (Tejada, 2012), como prueba el creciente número de referencias al respecto en la literatura científica (Ion y Cano, 2012). Sin embargo, para los docentes, desarrollar y, sobre todo, evaluar las competencias transversales supone un verdadero reto porque dichas competencias requieren innovaciones relevantes en las prácticas de enseñanza/aprendizaje, en los procedimientos de evaluación y en la propia organización escolar (Halász y Michel, 2011). Por ello, algunos autores han propuesto diferentes alternativas para evaluar las competencias transversales. Este es, por ejemplo, el caso de Solanes et al.(2008), quienes proponen un cuestionario como herramienta de evaluación de las mismas; cuestionario que se ha empleado como punto de partida en nuestro trabajo empírico.

\section{METODOLOGÍA DE INVESTIGACIÓN}

El estudio empírico que aquí presentamos se centra en el análisis de las percepciones del alumnado del GIDIDP del Centro Universitario de Mérida de la Universidad de Extremadura. Se decidió estudiar esta titulación porque generalmente sus egresados intentan autoemplearse o buscar empleo en la empresa privada, y de acuerdo con Corominas (2001), la empresa privada otorga más importancia a las competencias transversales y las cualidades personales de los candidatos a un puesto $\mathrm{d}$ trabajoque la Administración Pública. El objetivo de este trabajo es conocer cuáles son las competencias que los estudiantes manifiestan tener desarrolladas en mayor medida, así como analizar si la percepción sobre el nivel de competencias transversales de los estudiantes de GIDIDP es más positiva en segundo curso que en primero. 
Tabla 1: Competencias transversales (adaptado de Rico et al., 2013)

\begin{tabular}{|c|c|c|}
\hline Categoría & & Competencia \\
\hline \multirow[t]{11}{*}{ Instrumental } & Cognitiva & $\begin{array}{l}\text { Pensamiento: analítico, sistemático, reflexivo, lógico, crítico, creativo, } \\
\text { práctico, pensamiento deliberativo y colegial. }\end{array}$ \\
\hline & Metodológica & Organización del tiempo \\
\hline & & Resolución de problemas \\
\hline & & Toma de decisiones \\
\hline & & Planificación \\
\hline & & Aprendizaje continuo \\
\hline & Tecnológica & Base de datos \\
\hline & & Ordenador como una herramienta de trabajo \\
\hline & Lingüística & Comunicación oral \\
\hline & & Comunicación escrita \\
\hline & & Competencia en una lengua extranjera \\
\hline \multirow[t]{8}{*}{ Interpersonal } & Individual & Auto-motivación \\
\hline & & Persistencia y habilidad para adaptarse \\
\hline & & Comportamiento ético \\
\hline & Social & Diversidad y multiculturalismo \\
\hline & & Trabajo en equipo \\
\hline & & Comunicación interpersonal \\
\hline & & Resolución de conflictos \\
\hline & & Negociación \\
\hline \multirow[t]{9}{*}{ Sistemática } & Capacidad & Creatividad \\
\hline & emprendedora & Capacidad emprendedora \\
\hline & & Capacidad para innovar \\
\hline & Organización & Gestión por objetivos \\
\hline & & Gestión por proyectos \\
\hline & & Desarrollo de la calidad \\
\hline & Liderazgo & Capacidad de influencia \\
\hline & & Capacidad para delegar \\
\hline & Logro/Éxito & Orientación al logro \\
\hline
\end{tabular}

Con esta intención se aplicó un cuestionario que había sido previamente utilizado por Solanes et al. (2008). Se trata de un cuestionario de 45 preguntas cerradas, todas ellas siguiendo un formato de respuesta escala Likert de 5 puntos, siendo 1 la puntuación más baja, y 5 la más alta. La validez del instrumento, entendida como la capacidad de éste para medir lo que realmente quiere medir, se garantiza por la aplicación previa del mismo en el estudio de sus diseñadores. Y para verificar su fiabilidad se utilizó el alfa de Cronbach, cuyo valor $(0,95)$ nos indica que nuestro instrumento es fiable.

Nuestra población de estudio eran los estudiantes del Centro Universitario de Mérida de la Universidad de Extremadura que estaban matriculados en el GIDIDP, y concretamente aquellos estudiantes que estaban matriculados en el primer curso de la titulación durante el año académico 2010/2011 y en segundo curso durante el curso académico 2011/2012. En total, nuestra población objeto de estudio eran 38 estudiantes, siendo nuestra muestra de 13 alumnos, lo cual significa que se ha obtenido una tasa de respuesta del $34,21 \%$. Dado que se dio libertad al alumnado para responder o no al cuestionario, algunos de los estudiantes optaron por no hacerlo, y la mayoría sólo lo completó en una de las dos ocasiones, lo cual no impedía analizar si habían o no mejorado su percepción respecto a las competencias transversales que poseían, por lo que no fueron incluidos en el estudio. Es decir, sólo fueron considerados en nuestro análisis las respuestas de quienes habían respondido el cuestionario los dos años, de forma que los 13 estudiantes que respondieron el cuestionario el segundo año eran los mismos 13 estudiantes que lo habían respondido un año antes.

El estudio que aquí se presenta es una investigación longitudinal realizada en dos años consecutivos con el objetivo de analizar la evolución en la percepción de los estudiantes sobre su nivel de competencias. Para ello se aplicó el mismo cuestionario al mismo grupo de estudiantes, en dos momentos diferentes, con un curso académico de diferencia. Así, el cuestionario se administró por primera vez al finalizar el segundo semestre del curso académico 2010/2011, y por segunda vez al finalizar el curso 2011/2012. 
Para el análisis de los datos se realizó, en primer lugar, un estudio descriptivo de las variables a través de estadísticos de tendencia central y dispersión (media y desviación típica) con la intención de determinar cuáles eran las capacidades más o menos valoradas por el alumnado. Para realizar este análisis se utilizó el Statistical Package for the Social Sciences (SPSS), versión 19.0 para Windows. En segundo lugar se realizó un análisis comparativo que en nuestra investigación fue posible por pasar el cuestionario a una misma muestra, pero en dos momentos de sus vidas. Atendiendo a los resultados del test de bondad de ajuste de Kolmogorov-Smirnov no se verifica el supuesto de normalidad exigido para la aplicación de un contraste paramétrico basado en el estadístico t. Y dado el reducido tamaño de la muestra, se ha considerado una solución no paramétrica adecuada aproximarnos al problema a través del Bootstrap, posiblemente el método de remuestreo (los métodos de remuestreo consisten en generar un elevado número de muestras como base para determinar el comportamiento de determinados estadísticos) más conocido y más comúnmente utilizado, a pesar de su escasa presencia en los estudios de educación. Este método parte de los datos de la muestra original, y a partir de ellos genera nuevas muestras que sirven de base para estimar, de forma inductiva, la forma de distribución de los estadísticos, en lugar de partir de una distribución teórica asumida previamente (Gil, 2005). Para la implementación de este método de remuestreo se ha utilizado el software Resampling Stats Add-In for Excel.

\section{RESULTADOS}

Para conocer la opinión del alumnado del GIDIDP sobre el nivel de competencias transversales desarrollado se parte de un análisis descriptivo de los ítems incluidos en el cuestionario (tabla 2).

Tabla 2: Estadísticos descriptivos de todos los ítems del cuestionario

\begin{tabular}{|c|c|c|c|c|}
\hline & \multicolumn{2}{|c|}{$2010 / 2011$} & \multicolumn{2}{|c|}{$2011 / 2012$} \\
\hline Ítem & Media & $\begin{array}{c}\text { Desviación } \\
\text { Típica }\end{array}$ & Media & $\begin{array}{c}\text { Desviación } \\
\text { Típica }\end{array}$ \\
\hline 1. Conocimientos de la carrera & 2,91 & 0,944 & 3,23 & 0,599 \\
\hline 2. Capacidad de concentración para el estudio & 3,08 & 1,188 & 3,54 & 0,967 \\
\hline 3. Capacidad de innovación en las prácticas o trabajos & 2,77 & 1,166 & 3,31 & 0,947 \\
\hline $\begin{array}{l}\text { 4. Capacidad para valorar los resultados académicos en } \\
\text { tus exámenes }\end{array}$ & 3,08 & 1,320 & 3,23 & 0,725 \\
\hline 5. Capacidad de adaptación a nuevas situaciones & 3,31 & 1,032 & 3,54 & 1,127 \\
\hline 6. Capacidad de previsión y planificación & 3,08 & 1,038 & 3,38 & 1,121 \\
\hline 7. Capacidad de organizar tu tiempo de estudio y trabajo & 2,62 & 1,193 & 3,38 & 0,961 \\
\hline 8. Capacidad para organizar equipos de trabajo & 2,85 & 0,801 & 2,92 & 0,862 \\
\hline $\begin{array}{l}\text { 9. Capacidad de aprovechamiento óptimo de los } \\
\text { recursos propios }\end{array}$ & 3,08 & 1,320 & 3,38 & 0,870 \\
\hline $\begin{array}{l}\text { 10. Capacidad de aprovechamiento óptimo de los } \\
\text { recursos de la Universidad }\end{array}$ & 2,46 & 1,050 & 2,69 & 1,032 \\
\hline $\begin{array}{l}\text { 11. Capacidad de negociación en asuntos de clase } \\
\text { buscando el mejor logro }\end{array}$ & 3,08 & 1,256 & 3,23 & 1,013 \\
\hline $\begin{array}{l}\text { 12. Capacidad de resolución de problemas en materias } \\
\text { de clase, en las prácticas, etc. }\end{array}$ & 3,08 & 1,115 & 3,46 & 0,519 \\
\hline 13. Capacidad de síntesis & 3,38 & 1,387 & 3,62 & 1,261 \\
\hline 14. Capacidad para ser competitivo/a & 2,77 & 1,166 & 3,54 & 0,877 \\
\hline $\begin{array}{l}\text { 15. Capacidad para mantener el rendimiento habitual en } \\
\text { situaciones adversas o conflictivas }\end{array}$ & 2,54 & 1,127 & 2,85 & 1,144 \\
\hline $\begin{array}{l}\text { 16. Capacidad para actuar con desenvoltura y firmeza en } \\
\text { situaciones de tensión }\end{array}$ & 2,54 & 0,877 & 2,85 & 0,899 \\
\hline 17. Capacidad para trabajar en equipo & 3,46 & 0,967 & 3,08 & 0,862 \\
\hline $\begin{array}{l}\text { 18. Capacidad de coordinación en las prácticas o } \\
\text { trabajos }\end{array}$ & 3,23 & 1,092 & 3,23 & 1,092 \\
\hline
\end{tabular}


Tabla 2 (Continuación)

\begin{tabular}{|c|c|c|c|c|}
\hline & $2010 / 2011$ & $2011 / 2012$ & & \\
\hline Ítem & Media & $\begin{array}{l}\text { Desviación } \\
\text { Típica }\end{array}$ & Media & $\begin{array}{l}\text { Desviación } \\
\text { Típica }\end{array}$ \\
\hline $\begin{array}{l}\text { 19. Voluntariedad en el trabajo en equipo y en las } \\
\text { prácticas }\end{array}$ & 2,92 & 1,115 & 3,46 & 1,050 \\
\hline $\begin{array}{l}\text { 20. Capacidad y/o facilidad para relacionarte con tus } \\
\text { compañeros/as }\end{array}$ & 3,54 & 967 & 3,77 & 1,166 \\
\hline 21. Capacidad de comunicación & 3,08 & 1,256 & 3,31 & 1,109 \\
\hline $\begin{array}{l}\text { 22. Capacidad para las relaciones interpersonales } \\
\text { (empatía y escucha activa) }\end{array}$ & 3,08 & 1,256 & 3,38 & 1,261 \\
\hline $\begin{array}{l}\text { 23. Capacidad para relacionarte fácilmente con tus } \\
\text { profesores }\end{array}$ & 3,15 & 1,214 & 3,38 & 1,325 \\
\hline 24. Capacidad para ser emprendedor & 3,42 & 1,165 & 2,92 & 1,038 \\
\hline $\begin{array}{l}\text { 25. Capacidad para que los demás estudiantes acepten } \\
\text { tus ideas y propuestas }\end{array}$ & 3,08 & 1,115 & 3,15 & 1,144 \\
\hline $\begin{array}{l}\text { 26. Capacidad para inspirar confianza entre los } \\
\text { compañeros/as }\end{array}$ & 3,54 & 1,198 & 3,38 & 1,121 \\
\hline $\begin{array}{l}\text { 27. Capacidad de persuasión y obtención de ventajas sin } \\
\text { provocar hostilidades }\end{array}$ & 3,17 & 1,115 & 3,23 & 1,092 \\
\hline 28. Capacidad para aceptar nuevos cargos & 3,00 & 1,000 & 3,31 & 1,109 \\
\hline 29. Capacidad de relación con los demás compañeros/as & 3,23 & 1,536 & 3,46 & 0,967 \\
\hline $\begin{array}{l}\text { 30. Te proporciona acusada satisfacción la posibilidad } \\
\text { de dirigir personas y recursos }\end{array}$ & 2,75 & 1,138 & 3,23 & 1,301 \\
\hline $\begin{array}{l}\text { 31. Capacidad para proyectar buena imagen de la } \\
\text { Universidad en el exterior }\end{array}$ & 3,33 & 0,866 & 3,00 & 1,155 \\
\hline $\begin{array}{l}\text { 32. Capacidad de conocer tus propias características } \\
\text { personales y profesionales }\end{array}$ & 3,73 & 1,104 & 3,54 & 0,776 \\
\hline $\begin{array}{l}\text { 33. Capacidad para mantener la estabilidad y el equilibrio } \\
\text { en situaciones de tensión, adversas o con límite de } \\
\text { tiempo. }\end{array}$ & 2,85 & 1,144 & 2,85 & 0,801 \\
\hline $\begin{array}{l}\text { 34. Autoconfianza: confianza del estudiante en sus } \\
\text { capacidades potenciales y en la puesta en práctica de } \\
\text { sus conocimientos de la titulación. }\end{array}$ & 3,15 & 1,068 & 3,23 & 0,927 \\
\hline 35. Motivación por el estudio & 3,50 & 1,446 & 3,31 & 1,109 \\
\hline $\begin{array}{l}\text { 36. Capacidad para mantener el rendimiento habitual en } \\
\text { situaciones adversas o con límite de tiempo }\end{array}$ & 2,69 & 1,182 & 2,85 & 0,899 \\
\hline $\begin{array}{l}\text { 37. Capacidad para actuar con desenvoltura en las } \\
\text { exposiciones públicas }\end{array}$ & 2,38 & 1,044 & 3,00 & 0,913 \\
\hline $\begin{array}{l}\text { 38. Capacidad para sentir atracción por conseguir los } \\
\text { objetivos marcados }\end{array}$ & 3,69 & 1,251 & 4,00 & 0,913 \\
\hline $\begin{array}{l}\text { 39. Capacidad para aceptar con facilidad nuevas } \\
\text { responsabilidades }\end{array}$ & 3,00 & 1,080 & 3,69 & 0,947 \\
\hline 40. Resultados de los últimos exámenes & 3,54 & 1,127 & 3,15 & 0,987 \\
\hline $\begin{array}{l}\text { 41. Capacidad para encontrar soluciones nuevas y } \\
\text { originales en el trabajo (remunerado o no) }\end{array}$ & 2,80 & 1,135 & 3,08 & 0,760 \\
\hline $\begin{array}{l}\text { 42. Capacidad y facilidad para adaptarse a las nuevas } \\
\text { situaciones }\end{array}$ & 3,25 & 0,866 & 3,31 & 1,182 \\
\hline $\begin{array}{l}\text { 43. Capacidad de establecer sistemas para el } \\
\text { aprovechamiento óptimo de los recursos }\end{array}$ & 3,08 & 0,954 & 2,85 & 0,899 \\
\hline $\begin{array}{l}\text { 44. Capacidad para superar cualquier dificultad sin } \\
\text { recurrir a tus superiores }\end{array}$ & 3,15 & 0,987 & 3,31 & 0,947 \\
\hline $\begin{array}{l}\text { 45. Capacidad para desempeñar otro puesto de trabajo } \\
\text { diferente del que me estoy formando }\end{array}$ & 3,92 & 0,862 & 3,92 & 0,760 \\
\hline
\end{tabular}

Según los resultados del análisis descriptivo, cabe indicar que la valoración de las competencias que hacen los estudiantes de primer curso oscila entre 2,38 y 3,92, siendo la percepción media del nivel de competencia transversal de quienes estudian primer curso del GIDIDP de casi 3,13 puntos (ligeramente por 
encima del punto medio de la escala), situándose la desviación en torno a la media en el valor 0,81 . Estos mismos estudiantes, un curso más tarde, consideran que su nivel competencial transversal oscila entre 2,69 y 4, y que la percepción media se ha incrementado hasta los 3,31 puntos, disminuyendo la desviación típica hasta los 0,46 puntos.

Y dado que uno de los objetivos de este trabajo es investigar la evolución del nivel de competencias transversales adquirido por los estudiantes de segundo curso frente a los estudiantes de primero, en la figura 1 se recoge la valoración media de los 45 ítems o capacidades recogidas en el cuestionario, tanto para el curso académico 2010/2011, como para el curso 2011/2012.

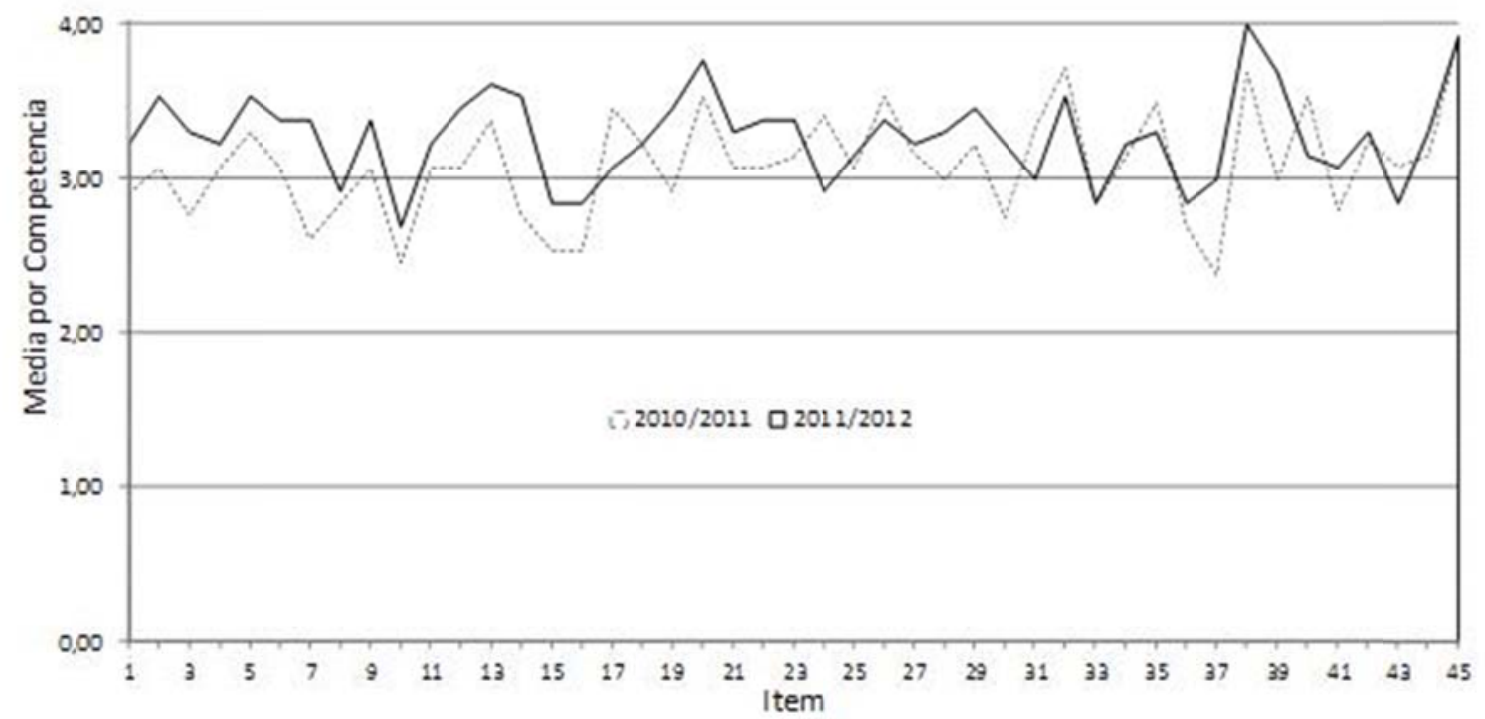

Fig. 1: Competencia transversal media del alumnado de GIDIDP de primer y segundo curso

De acuerdo con los datos que arroja el análisis descriptivo, durante el curso 2010/2011, las capacidades que las personas encuestadas manifestaban tener desarrolladas en mayor medida eran la capacidad para desempeñar otro puesto de trabajo diferente de aquel para el que se estaban formando, la capacidad de conocer las propias características personales y profesionales, y la capacidad de sentir atracción por conseguir los objetivos marcados (correspondientes a los ítems 45, 32 y 38). Y las capacidades que el alumnado manifestaba tener desarrolladas en menor medida eran las correspondientes a los ítems 37, 10, 15 y 16: capacidad para actuar con desenvoltura en las exposiciones públicas, capacidad de aprovechamiento óptimo de los recursos de la Universidad, capacidad para mantener el rendimiento habitual en situaciones adversas o conflictivas, y capacidad para actuar con desenvoltura y firmeza en situaciones de tensión.

Un curso después, el alumnado consideraba que las competencias que tenían más desarrolladas eran las correspondientes a los ítems 38, 45 y 20: capacidad para sentir atracción por conseguir los objetivos marcados, capacidad para desempeñar otro puesto de trabajo diferente de aquel para el que se están formando, y capacidad para relacionarse fácilmente con sus compañeros. $Y$ las que consideraban tener menos desarrolladas eran la capacidad de aprovechamiento óptimo de los recursos de la Universidad (ítem 10), seguidas de la capacidad para mantener el rendimiento habitual en situaciones adversas o conflictivas; la capacidad para actuar con desenvoltura y firmeza en situaciones de tensión; la capacidad para mantener la estabilidad y el equilibrio en situaciones de tensión, adversas o con límite de tiempo; la capacidad para mantener el rendimiento habitual en situaciones adversas o con límite de tiempo; y la capacidad para establecer sistemas para el aprovechamiento óptimo de los recursos (ítems 15, 16, 33, 36 y 43).

Entre un curso académico y otro mejora la percepción del nivel de desarrollo de muchos de las capacidades o ítems estudiados, siendo la mejora especialmente notable en los ítems 14, 7, y 39, correspondientes a la capacidad para ser competitivo, la capacidad para organizar el tiempo de estudio y trabajo, y la capacidad para aceptar con facilidad nuevas responsabilidades. Sin embargo, la percepción que el alumnado tiene del grado de desarrollo de ciertas competencias empeora entre el primer y el segundo curso, siendo la diferencia de medias especialmente notable para los ítems 24, 40 y 17: capacidad para ser emprendedor, resultados de los últimos exámenes del estudiante, y capacidad para trabajar en equipo. 
Antes de llevar a cabo el contraste de la hipótesis de que el nivel competencial transversal del alumnado de segundo curso es superior al del alumnado de primer curso, se procedió a realizar la prueba de bondad del ajuste. Para ello se emplearon los test de Kolmogorov-Smirnov y de Shapiro-Wilks, constatándose que los datos no cumplían el supuesto de normalidad, lo cual nos llevó a emplear pruebas no paramétricas a través y a utilizar un método de remuestreo, Bootstrap (tabla 3).

Tabla 3: Test de Normalidad

\begin{tabular}{ccccccc}
\hline & \multicolumn{3}{c}{ Kolmogorov-Smirnov $^{\mathrm{a}}$} & \multicolumn{3}{c}{ Shapiro-Wilk } \\
\hline & Estadístico & G.L. & Sig. & Estadístico & G.L. & Sig. \\
\hline Media 1 $^{\circ}$ & 0,256 & 13 & 0,019 & 0,862 & 13 & 0,040 \\
\hline Media 2 $^{\circ}$ & 0,93 & 13 & $0,200^{*}$ & 0,975 & 13 & 0,945 \\
\hline
\end{tabular}

${ }^{a}$ Corrección de significación de Lilliefors

* Este es un límite inferior de la significación verdadera.

Ante muestras tan pequeñas, la suposición de normalidad parece arriesgada. $Y$ dado que los procedimientos basados en Bootstrap implican obviar los supuestos sobre la distribución teórica que siguen los estadísticos (Gil, 2005, p. 252), y permiten obtener resultados correctos incluso con muestras pequeñas de entre 10 y 20 casos (Bickel y Krieger, 1989), se ha optado por emplear el método Bootstrap para estudiar el modo en que se distribuye el estadístico en un conjunto elevado de muestras generado por remuestreo. Para hacerlo, se han seguido los siguientes pasos. Primero, se han combinado los datos disponibles de los grupos de estudiantes de primer y segundo curso del GIDIDP, obteniendo un conjunto $x=\{z, y\}$ que consta de un total de 26 valores, denominando $z^{*}$ a los 13 primeros valores e $y^{*}$ a los 13 restantes. A continuación, para la muestra $\mathrm{x}^{*}$, se calcula la diferencia de medias para cada ítem, ya que dicha diferencia será el estadístico en el que se base el contraste de hipótesis. Se han repetido los pasos anteriores 5000 veces, ya que según Chernick (1999) esta es una cifra adecuada para la mayoría de los problemas de investigación planteados. Y finalmente, se ha construido la distribución de frecuencias para al estadístico diferencia de medias para cada ítem.

Como resultado de todos los pasos anteriormente mencionados, seguidos para todos y cada uno de los ítems de nuestro cuestionario, se han encontrado diferencias significativas en tres de los ítems. Así, en la tabla 4 se recoge la distribución de frecuencias de valores del ítem 7 (capacidad para organizar tu tiempo de estudio o trabajo). Dichas frecuencias, obtenidas a través de Bootstrap, constituyen una estimación de la distribución muestral para el estadístico diferencia de medias.

Tabla 4: Capacidad para organizar el tiempo de estudio/trabajo (distribución de frecuencias)

\begin{tabular}{|c|c|c|c|}
\hline & Frecuencia absoluta & Frecuencia relativa & Frecuencia relativa acumulada \\
\hline$-0,77$ & 13 & 0,3 & 0,3 \\
\hline$-0,62$ & 129 & 2,6 & 2,8 \\
\hline$-0,46$ & 424 & 8,5 & 11,3 \\
\hline$-0,31$ & 687 & 13,7 & 25,1 \\
\hline$-0,15$ & 826 & 16,5 & 41,6 \\
\hline 0,00 & 871 & 17,4 & 59,0 \\
\hline 0,15 & 809 & 16,2 & 75,2 \\
\hline 0,31 & 650 & 13,0 & 88,2 \\
\hline 0,46 & 432 & 8,6 & 96,8 \\
\hline 0,62 & 141 & 2,8 & 99,6 \\
\hline 0,77 & 18 & 0,4 & 100,0 \\
\hline Total & 5000 & 100,0 & \\
\hline
\end{tabular}

La diferencia de medias en el ítem 7 en la muestra original es de 0,62. Contando el número de veces que la diferencia de medias supera o iguala a dicho valor, se obtiene la frecuencia relativa, es decir, la probabilidad de encontrar, aceptando la hipótesis nula como cierta, una diferencia de medias igual o mayor a la observada. Así, partiendo de la distribución empírica, 159 de las 5000 muestras han dado lugar a diferencias iguales o mayores que a dicho valor. Por tanto, la probabilidad que buscamos resulta ser $p=$ 0,0318 . Y puesto que se trabaja con un nivel de confianza del $5 \%(\alpha=0,05)$, puede afirmarse que existe una diferencia significativa a favor del grupo de estudiantes de segundo curso, por lo que la capacidad para organizar el tiempo de estudio y/o trabajo es mejor entre el alumnado de segundo curso que entre el alumnado de primero. 
En la tabla 5 se recoge la estimación de la distribución de frecuencias muestral, obtenida a partir de Bootstrap, para el estadístico diferencia de medias, del ítem 14 (capacidad para ser competitivo/a). La diferencia de medias de dicho ítem en la muestra original es de 0,77. Partiendo de la distribución empírica, 131 de las 5000 muestras han dado lugar a diferencias iguales o mayores a dicho valor, siendo la probabilidad relativa $p=0,0262$. Asumiendo un nivel de confianza del $5 \%(\alpha=0,05)$, cabe decir que la capacidad para ser competitivo/a del alumnado de segundo curso es superior a la del alumnado de primer curso, siendo dicha diferencia estadísticamente significativa.

Tabla 5: Capacidad para ser competitivo/a (distribución de frecuencias)

\begin{tabular}{|c|c|c|c|}
\hline & Frecuencia absoluta & Frecuencia relativa & $\begin{array}{c}\text { Frecuencia relativa } \\
\text { acumulada }\end{array}$ \\
\hline$-0,92$ & 19 & 0,4 & 0,4 \\
\hline$-0,77$ & 111 & 2,2 & 2,6 \\
\hline$-0,62$ & 297 & 5,9 & 8,5 \\
\hline$-0,46$ & 439 & 8,8 & 17,3 \\
\hline$-0,31$ & 499 & 10,0 & 27,3 \\
\hline$-0,15$ & 721 & 14,4 & 41,7 \\
\hline 0,00 & 870 & 17,4 & 59,1 \\
\hline 0,15 & 711 & 14,2 & 73,3 \\
\hline 0,31 & 509 & 10,2 & 83,5 \\
\hline 0,46 & 406 & 8,1 & 91,6 \\
\hline 0,62 & 287 & 5,7 & 97,4 \\
\hline 0,77 & 118 & 2,4 & 99,7 \\
\hline 0,92 & 13 & 0,3 & 100,0 \\
\hline Total & 5000 & 100,0 & \\
\hline
\end{tabular}

La tabla 6 recoge la estimación de la distribución de frecuencias de la muestra, obtenida a partir del Bootstrap, para intervalos de valores del ítem 37 (capacidad para actuar con desenvoltura en exposiciones públicas). La diferencia de medias en la muestra original es de 0,62. De las 5000 muestras, 134 han dado lugar a diferencias iguales o mayores que dicho valor. Por tanto, $p=0,0268$. Asumiendo un nivel de confianza del $5 \%(\alpha=0,05)$, se detecta una diferencia significativa entre la capacidad para actuar con desenvoltura en exposiciones públicas de los estudiantes de segundo curso y la de los estudiantes de primero, estando dicha capacidad más desarrollada en el alumnado del curso de nivel superior.

Tabla 6: Capacidad para actuar con desenvoltura en exposiciones públicas (distribución de frecuencias)

\begin{tabular}{|c|c|c|c|}
\hline & Frecuencia absoluta & $\begin{array}{c}\text { Frecuencia } \\
\text { relativa }\end{array}$ & $\begin{array}{c}\text { Frecuencia relativa } \\
\text { acumulada }\end{array}$ \\
\hline$-0,77$ & 24 & 0,5 & 0,5 \\
\hline$-0,62$ & 117 & 2,3 & 2,8 \\
\hline$-0,46$ & 324 & 6,5 & 9,3 \\
\hline$-0,31$ & 610 & 12,2 & 21,5 \\
\hline$-0,15$ & 915 & 18,3 & 39,8 \\
\hline 0,00 & 1045 & 20,9 & 60,7 \\
\hline 0,15 & 867 & 17,3 & 78,0 \\
\hline 0,31 & 641 & 12,8 & 90,9 \\
\hline 0,46 & 323 & 6,5 & 97,3 \\
\hline 0,62 & 120 & 2,4 & 99,7 \\
\hline 0,77 & 14 & 0,3 & 100,0 \\
\hline Total & 5000 & 100,0 & \\
\hline
\end{tabular}

En general los sujetos de segundo curso tienden a mostrar una mejor percepción de su nivel de competencias (tabla 2). Sin embargo, el escaso número de sujetos disponibles para realizar el contraste provoca que tales diferencias no lleguen a ser significativas $(p>0,05)$, por lo que nuestra hipótesis sólo recibe apoyo de forma parcial. Estos resultados avalan, en cierta medida, la hipótesis planteada, pero futuros estudios deberán contrastar dicha hipótesis en muestras más amplias.

\section{DISCUSIÓN}


Independientemente de los resultados obtenidos, la aportación del presente estudio se basa principalmente en la necesidad de establecer herramientas y metodologías para enseñar y evaluar al alumnado universitario de forma integral, en todas las competencias que conforman el plan de estudio, considerando las competencias transversales como parte fundamental del proceso de formación. En este sentido, las mejoras que el alumnado percibe en el nivel de desarrollo de ciertas competencias coinciden fundamentalmente con el trabajo realizado en clase y de forma más específica en las sesiones de tutorías programadas (ECTS). Gran parte de los docentes de la titulación enfocó el contenidos de dichas sesiones en la realización de actividades y trabajos conducentes al desarrollo de competencias transversales, cuya asimilación se evaluaba mediante exposiciones en el aula, progresando en habilidades para hablar en público y despertando entre el alumnado cierto grado de competitividad. La gestión del tiempo y el trabajo continúo fueron también dos consecuencias significativas del trabajo realizado en las tutorías programadas. Igualmente se considera que los resultados menos positivos obtenidos en el desarrollo de competencias tales como capacidad para mantener el equilibrio en situaciones de tensión o la dificultad para gestionar la presión derivada de restricciones temporales podrían deberse a cierta descoordinación del equipo docente, falta de coordinación que pudo producirse al trabajar en profundidad ciertas competencias en detrimento de otras igualmente importantes.

Más en detalle, destacamos que durante el curso 2010/2011 entre las competencias que manifiestan tener desarrolladas en mayor medida destacan la predisposición para desempeñar ocupaciones y roles diferentes, la capacidad para interiorizar las características personales y profesionales y el empeño por conseguir los objetivos marcados (correspondientes a los ítems 45, 32 y 38). Asimismo, entre las capacidades que el alumnado considera tener desarrolladas en menor medida subrayamos las correspondientes a los ítems 37, 10, 15 y 16: competencias para actuar con desenvoltura en exposiciones públicas, aprovechamiento óptimo de los recursos de la Universidad, capacidad para mantener el rendimiento habitual en situaciones adversas o conflictivas, y capacidad para actuar con firmeza en situaciones de tensión.

Sin embargo, se aprecia como en el curso siguiente (2011-2012), el alumnado considera que las competencias que tienen más desarrolladas son las correspondientes a los ítems 38, 45 y 20: capacidad para sentir atracción por conseguir los objetivos marcados, versatibilidad para desempeñar ocupaciones diferentes y capacidad para relacionarse con sus compañeros. Por otra parte, las competencias que manifiestan tener menos desarrolladas podría resumirse en capacidad de aprovechamiento de los recursos de la Universidad (ítem 10), seguidas de las competencias necesarias para mantener el rendimiento habitual en situaciones adversas o conflictivas, para actuar con firmeza en situaciones de tensión y la capacidad para establecer sistemas de aprovechamiento óptimo de los recursos (ítems 15, 16, 33, 36 y 43).

Observamos cómo entre un curso académico y otro mejora la percepción del nivel de desarrollo de muchas de las capacidades o ítems estudiados, siendo la mejora especialmente notable en los ítems 14, 7, y 39, correspondientes a la capacidad para ser competitivo, competencias para gestionar el tiempo de estudio y trabajo y la capacidad para aceptar con facilidad nuevas responsabilidades. Sin embargo, encontramos también la percepción que el alumnado tiene de cierta disminución en el grado de desarrollo de ciertas competencias entre el primer y el segundo curso, siendo la diferencia especialmente notable en los ítems 24, 40 y 17: capacidad para ser emprendedor, resultados en las pruebas de certificación y capacidad para trabajar en equipo.

No obstante, y aunque en términos generales los sujetos de segundo curso tienden a mostrar una mejor percepción de su nivel de competencias transversales, el escaso número de participantes disponibles para realizar el contraste provoca que tales diferencias no lleguen a ser suficientemente significativas. En este sentido, a pesar de que los resultados avalan, en cierta medida, la hipótesis planteada, futuros estudios deberán contrastar dicha hipótesis en muestras más amplias.

\section{CONCLUSIONES}

El alumnado de segundo curso presenta una mejor percepción del desarrollo de las competencias transversales que el de primero, especialmente en las competencias de trabajo en equipo, conocimiento de las características personales y aptitudes profesionales y capacidad para conseguir los objetivos programados.

La principal aportación de este estudio consiste en emplear herramientas para evaluar al alumnado universitario de forma integral, considerando las competencias transversales como parte fundamental del proceso de formación. 


\section{REFERENCIAS}

Alonso, L.; C. Fernández y J. Nyssen, J. (Agencia Nacional de Evaluación de la Calidad y Acreditación), El debate sobre las competencias. Una investigación cualitativa en torno a la educación superior y el mercado (en línea), 2009: http://www.aneca.es/content/download/10356/115906/file/publi_competencias_090303.pdf Acceso: 10 de Febrero (2013).

Agencia Nacional de Evaluación de la Calidad y Acreditación (ANECA), Protocolo de evaluación para la Verificación de Títulos Universitarios Oficiales (Grado y Máster) (en línea), 2008: http://www.aneca.es/content/download/12387/153627/file/verifica_protocolo_gradomaster_110207.pdf.

Acceso: 20 de Enero (2013)

Bickel, P. J. y A. M. Krieger. Confidence Bands for a Distribution Function Using the Bootstrap, Journal of the American Statistical Association, 84 (405), 95-100 (1989).

Boyatzis, R. E., Competences in the 21st century, Journal of Management Development, 27 (1), 5-12 (2008).

Bridgstock, R. The graduate attributes we've overlooked: enhancing graduate employability through career management skills. Higher Education Research \& Development, 28 (1), 31-44 (2009).

Cajide, J., A. Porto, C. Abeal., F. Barreiro, E. Zamora, A. Expósito y J. Mosteiro. Competencias adquiridas en la Universidad y habilidades requeridas por los empresarios. Revista de Investigación Educativa, 20 (2), 449-467 (2002).

Chernick, M. R., Bootstrap methods: a practitioner's guide, Wiley \& Sons, Nueva York, Estados Unidos (1999).

Corominas, E., Competencias Genéricas en la Formación Universitaria, Revista de Educación, 325, 299-321 (2001).

Delors, J. (Organización de las Naciones Unidas para la Educación), La educación encierra un tesoro. Informe a la UNESCO de la Comisión Internacional sobre la Educación para el siglo XXI, UNESCO, París, Francia (1996).

Fernández, A. (coord.), Desarrollo y Evaluación de Competencias en Educación Superior. Narcea de Editores, Madrid, España (2009).

Gil, J., Aplicación del método Bootstrap al constraste de hipótesis en la investigación educativa, Revista de Educación, 336, 251-265 (2005)

González, J. y R. Wagenaar, Tuning eductional structures in Europe, Universidad de Deusto, Bilbao (2003).

Halász, G. y A. Michel, Key competences in Europe: interpretation, policy formulation and implementation, European Journal of Education, 46 (3), 289-306 (2011).

Ion, G. y E. Cano, La formación del profesorado universitario para la implementación de la evaluación por competencias, Educación XX1, 15 (2), 249-270 (2012).

Jiménez, L.; Ramos, F. J.; Ávila, M., Las Universidades Españolas y el EEES: Un estudio sobre los títulos de Grado de Maestro en Educación Primaria. Formación Universitaria, 5 (1), 33-44 (2012).

McClelland, D. C. , Testing for Competence Rather Than for "Intelligence", American Psychologist, 28 (1), 114 (1973).

Mulder, M.; J. Gulikers; H. Biemans y R. Wesselink, R., The new competence concept in higher education: error or enrichment?, Journal of European Industrial Tranining, 33 (8/9), 775-770 (2009).

Organización para la Cooperación y el Desarrollo Económico (OCDE). Investing in Competencies for all. Meeting of the OECD Education Ministers (communiqué) (en línea), 2008: http://www.oecd.org/dataoecd/48/24/1870589.pdf. Acceso: 31 de Enero (2013). 
Organización para la Cooperación y el Desarrollo Económico (OCDE). Definition and selection of competencies (DeSeCo) (en línea), 2011: http://www.oecd.org/dataoecd/47/61/35070367.pdf. Acceso: 31 de Enero (2013)

Ortega, M. C., Competencias emergentes del docente ante las demandas del EEES, Revista Española de Educación Comparada, 16, 305-327 (2013).

Ponsa, P., B. Amante, B.; J. A. Roman, S. Oliver, M. Díaz y J. Vives, Higher Education Challenges: Introduction of Active Methodologies in Engineering Curricula, International Journal of Engineering Education, 25 (4), 799-813 (2009).

R.D. 1393/2007. Gobierno de España, Real Decreto 1393/2007, de 29 de octubre, por el que se establece la ordenación de las enseñanzas universitarias oficiales, Boletín Oficial del Estado 260, de 30 de octubre, 44037-44038, España (2007)

Rico, M., J. Coppens, P. Ferreira, H. Sánchez, H. y J. E. Agudo, Everything Matters: Development of CrossCurricular Competences in Engineering Through Web 2.0 Social Objects, In Ubiquitous and Mobile Learning in the Digital Age by D. G. Sampson, P. Isaias, D. Ifenthaler, y J. M. Spector (Eds.), pp 139-157, Springer, Nueva York, Estados Unidos (2013).

Rychen, D. S. y Salganik, L. H., Definition and selection of key competences. En el Fourth General Assemply of the OECD Education Indicators Programme, The INES Compendium, Contributions from the INES Networks and Working Groups, pp. 61-74. Tokio, Japan: Organización para la Cooperación y el Desarrollo Económico (2000).

Solanes, A., R. Núñez y J. Rodríguez, Elaboración de un cuestionario para la evaluación de competencias genéricas en estudiantes universitarios. Apuntes de Psicología, 26 (1), 35-49 (2008).

Tejada, J., La evaluación de las competencias en contextos no formales: dispositivos e instrumentos de evaluación, Revista de Educación, 354, 731-745 (2011). 